\title{
Challenges Facing at Electron Microscopy Core Laboratory: Mitochondria Structural Preservation and 3D EM Data Presentation
}

Fengxia (Alice) Liang ${ }^{1}$, Chris Petzold ${ }^{1}$, Kristen Dancel-Manning ${ }^{1}$, Joseph Sall ${ }^{1}$, Patrick Ren ${ }^{2}$ and Chuxuan Zhou $^{3}$

${ }^{1}$ New York University Langone Health, New York, New York, United States, ${ }^{2}$ Stuyvesant High School, New York, New York, United States, ${ }^{3}$ Concord Academy, Concord, Massachusetts, United States

Modern electron microscopy allows scientists to view cells and tissues in great details from two-dimensional to three-dimensional in large scale. However, optimal ultrastructural preservation of various biological samples to achieve highest resolution with desired microscope remains a challenge. Centralized electron microscopy resources capitalize on the coequal magnifying and resolving power of the microscopes while relying on the technical competence of specialists, for training, equipment maintenance and guidance in preparatory procedures. Working in a research-driven microscopy core laboratory, we endeavor to understand the specific objectives of each assigned research project, identify suitable instrumentation and protocols that offer best and assist the interpretation of the data. Such an approach allows us to work with researches from variety of disciplines, including material, chemical and biological sciences.

Mitochondria are metabolic organelles that actively transform their ultrastructure involved in antiviral responses, and cell death. The integrity of the mitochondrial membrane is crucial for maintaining the mitochondria membrane potentials which drive ATP production and mitochondria homeostasis. Preservation of mitochondria structure is essential to the quantitative measurement of mitochondria fusion or fragmented dynamics, which might link to cellular metabolism in cancer research. Although glutaraldehyde-osmium fixative is often used to preserve the overall cell morphology, it is not optimal for preserving mitochondria of different kinds of cells or tissues. We found that decreasing dehydration time and performing most processing at $4{ }^{\circ} \mathrm{C}$ greatly improves mitochondria inner membrane preservation of Drosophila ovary[1], especially for germ cells. We also demonstrated that reduced osmium can greatly enhance the contrast of mitochondria membrane in several cultured cell lines (Fig. 1). In addition, a modified rOTO protocol (rOTO: reduced osmium-thiocarbohydrzide-osmium), with additional uranyl acetate and lead aspartate en bloc staining, further increase heavy-metal content $[2,3,4]$, such that the mitochondria membrane structure of mouse T cell is greatly highlighted (Fig. 2), as well as in vivo mouse urothelium (data not show). Such an improved membrane contrast allowed us to discover the intro-mitochondria lipid droplet formation in the Snx31-knock out mouse urothelium, which may serve as a detoxification mechanism to sequester/remove excess uroplakins, the major components on the apical surface of urothelium forming 2D crystals hexagonally packed 16-nm particles, that would otherwise form toxic insoluble protein aggregates [5]. On the other hand, mitochondria structure preservation in tissues, especially skeleton muscles would greatly rely on the successful operation of vascular perfusion in which fixative is delivered through the blood vessels (data not show).

With well-preserved biological samples, one can easily achieve high-resolution imaging in two or threedimension. However, three-dimensional electron microscopy generates a large volume of images which need to be translated into a presentable format that involves image segmentation and 3D model generation (Fig.2). With commercially available software, it is still a challenge for busy core facility staff. Giving the labor intensity and billing issues involved in image analysis, our view is the most efficient and economical way to achieve image analysis in the context of an imaging core facility, is to train project related graduate students or postdocs, and recruit volunteer high school or undergraduate students, guided by experienced core staff, to utilize commercially available software for segmentation, and generate final presentable 3D models. 


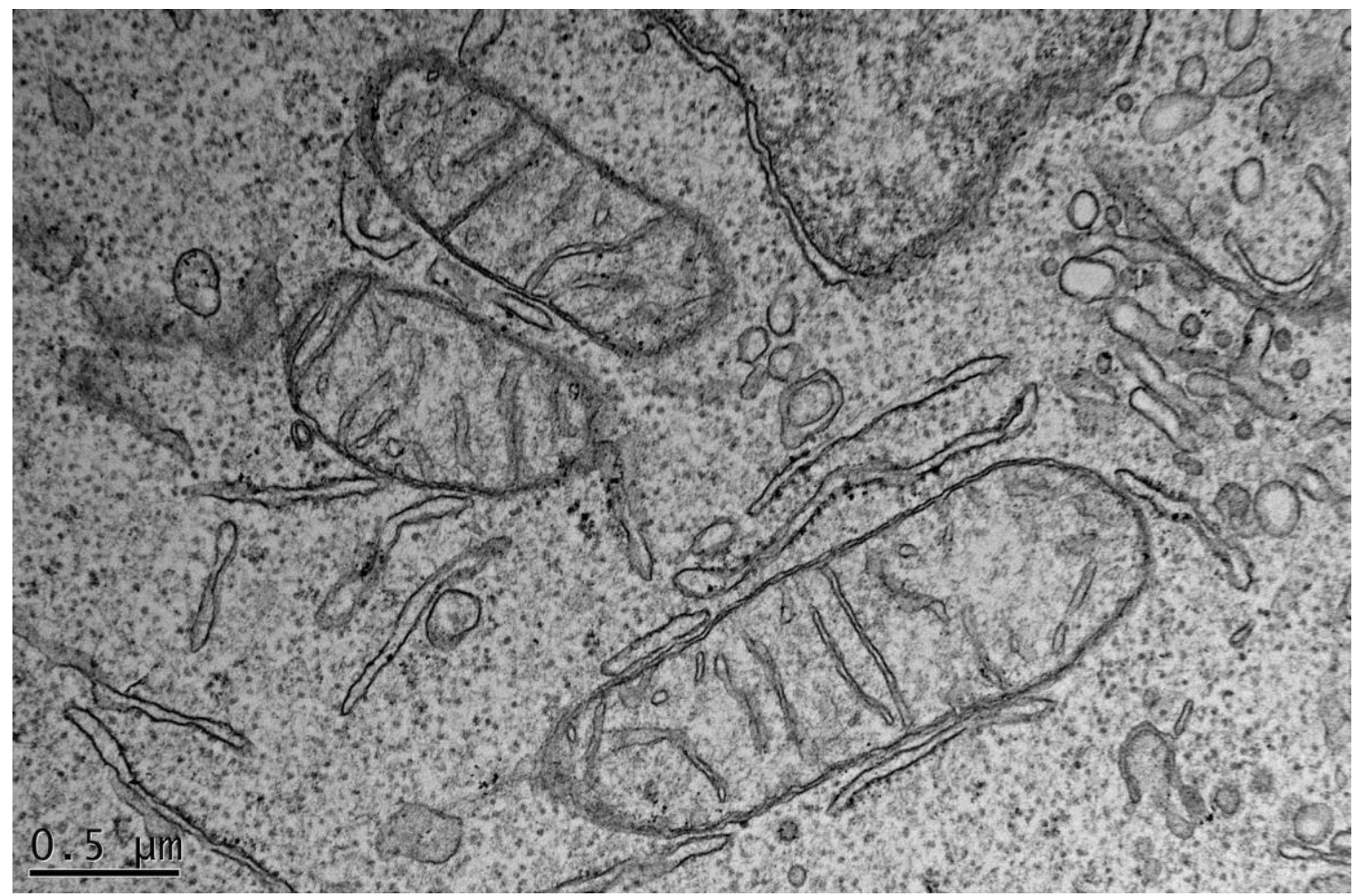

Figure 1. Reduced osmium can enhance membrane contrast of mitochondria in various in vitro cultured cell lines.
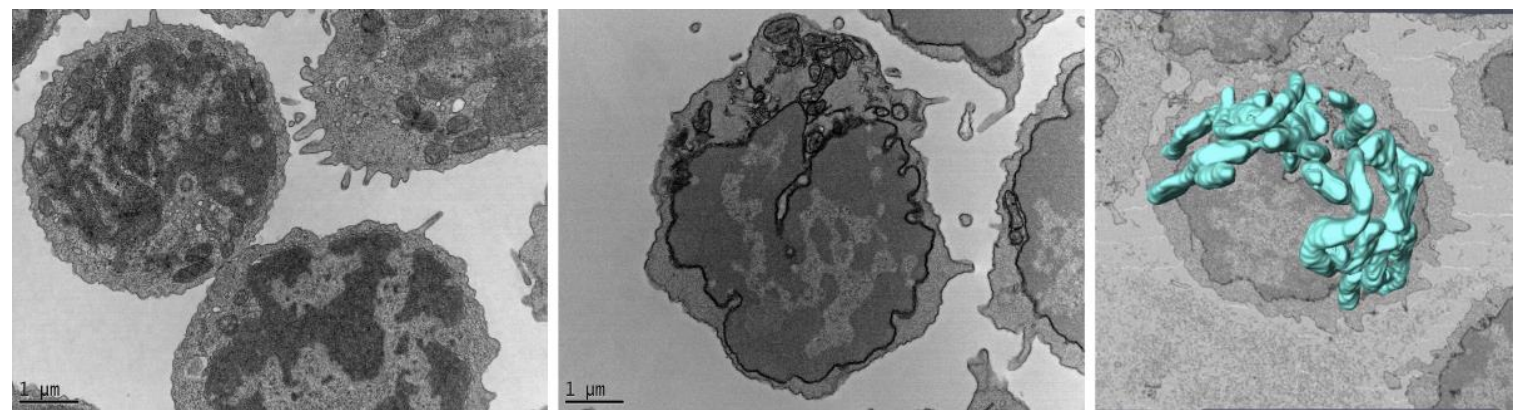

Figure 2. Different sample preparation methods reveal different mitochondria appearance in flow sorted mouse $\mathrm{T}$ cells, rOTO method greatly improved mitochondria membrane contrast which is essential for serial black-face SEM imaging.

\section{References}

[1] TR Hurd et al, Methods in Mol. Biol., (2015), 1328, p.151.

[2] AM Seligman et al, J. Cell Biol., (1966), 30 p.424.

[3] T Deerinck et al, Microsc. Microanal., (2010), 16, p.1138.

[4] JC Tapia et al, Nat. Protoc., (2012), 16, p.193.

[5] Y Liao et al, Mol Biol Cell, (2015), 30 (24), p.2969. 